\title{
Parasitism and over-wintering status of tachinids (Diptera) on larvae of the fall webworm, Hyphantria cunea Drury (Lepidoptera: Arctiidae), in the Kanto Region of Japan
}

\author{
Masahiko Watanabe* \\ Department of Physiology and Genetic Regulation, National Institute of Agrobiological Sciences (NIAS); Tsukuba, Ibaraki \\ 305-8634, Japan
}

(Received 20 October 2004; Accepted 2 February 2005)

\begin{abstract}
I investigated seasonal changes in the levels of parasitism and the over-wintering status of tachinids on the larvae of Hyphantria cunea. Six species of tachinids were found to parasitize populations of $H$. cunea at Tsukuba, in central Japan. The rate of parasitism by tachinids was extremely high in the third host generation, especially during the wandering stage. The parasitization rate by the three dominant species, Exorista japonica, Drino inconspicuoides and Compsilura concinnata, was always higher in the wandering larvae than in the feeding larvae. The over-wintering strategy of tachinids differed between species; $C$. concinnata over-winters at the maggot stage and E. japonica at the early instar maggot and puparial stages. In E. japonica, the rate of over-wintering survival differed greatly between the two over-wintering strategies; individuals over-wintering at the maggot stage within hosts almost all succeeded in adult eclosion in the next year, whereas those over-wintering at the puparial stage out of hosts often died early in the next spring. Many E. japonica maggots over-wintered within hosts collected in late October, suggesting that parasitizing host larvae in late autumn is advantageous for E. japonica. This study provides evidence of different parasitic strategies in tachinid species that use $H$. cunea as a host.
\end{abstract}

Key words: Tachinid; parasitism; overwintering; Hyphantria cunea

\section{INTRODUCTION}

The fall webworm, Hyphantria cunea Drury, is known as one of the most serious pests for many species of cultured and ornamental trees in the world (Warren and Tadic, 1970). The species distribution expanded to central Europe and eastern Asia in the 1940s (Umeya and Ito, 1977): it was first discovered in Tokyo in 1945 due to an accidental introduction from North America, and has since gradually expanded its distribution throughout most of Japan (Hasegawa, 1966; Masaki, 1975; Gomi, 1996). Webworm larvae are extremely polyphagous and attack at least 337 species of plants in Japan alone (Hasegawa, 1966).

$H$. cunea exhibited a bivoltine lifecycle during the first three decades after its invasion of Japan (Masaki, 1975). The present lifecycle has shifted from bivoltine to trivoltine in southwestern Japan
(Gomi, 1990, 1996). Gomi (1997) reported that bivoltine and trivoltine populations of $H$. cunea coexist in the Tsukuba region of central Japan $\left(36.0^{\circ} \mathrm{N}\right)$, where over-wintered pupae of both populations emerge as adults in May. Larvae of the first and second generations occur around June to July and August, respectively. Some individuals of the second generation enter pupal diapause and overwinter, and others eclose and produce the third generation without diapause. Individuals of the third generation always enter pupal diapause during October to November.

Larvae of $H$. cunea live in groups inside the nest-web only from the first to the third instars. Mortality is relatively low during the early larval stages due to the protective influence of the nestweb, but increases dramatically from the fourth to sixth or seventh instars, after leaving the web (Ito et al., 1968; Masaki, 1975). The main factors

*E-mail: masahiko@affrc.go.jp

DOI: 10.1303/aez.2005.293 
leading to high mortality are parasitization by parasitic wasps and flies, and predation by birds and predatory insects.

Many species of parasitic wasps and flies have been recorded for $H$. cunea not only in their native USA, but also in introduced areas: Europe, Korea and Japan (Hasegawa, 1966; Warren and Tadic, 1967; Kim, 1968). Globally, more than 40 species of tachinids have been reported as larval or larvalpupal parasitoids of $H$. cunea, 13 of which are found in Japan (Warren and Tadic, 1967; Shima, 1999). During the last few decades, however, there have only been a few field investigations of tachinids attacking $H$. cunea (Konno, 1998; Kan et al., 2003). Species composition and the parasitism rate of tachinids possibly change in association with the change in voltinism in $H$. cunea. Furthermore, over-wintering status is not clear in most species of tachinids. Such information is important for the evaluation of tachinids as potential biological control agents for $H$. cunea and to understand the survival strategies of tachinids. In the present study, I investigated seasonal changes in parasitism rate and over-wintering strategy in tachinid species using $H$. cunea as a host.

\section{MATERIALS AND METHODS}

Seasonal changes in tachinid parasitism. $H$. cunea produces two or three generations annually in Tsukuba, Ibaraki Prefecture, Japan (Gomi, 1996). Between July 1995 and October 1997, last instar larvae (6th or 7th instar) of each generation were collected from a row of Liquidambar styracciflua L. (about 500 plants on either side of a $3 \mathrm{~km}$ stretch of national road). Because of the low density of $H$. cunea in 1996, third generation larvae were collected mainly from trees of the same species at another site, about $1 \mathrm{~km}$ away from the above-mentioned national road. To determine the rate of tachinid parasitization, larvae collected in the field were given an artificial diet (Insecta LF, Nihon Nosan Kogyo, Yokohama) in a plastic container (upper diameter, $5 \mathrm{~cm}$; bottom diameter, $4 \mathrm{~cm}$; height, $5 \mathrm{~cm}$ ) under a $16 \mathrm{~h}$ light: $8 \mathrm{~h}$ dark (16L : 8D) photoperiod at $25^{\circ} \mathrm{C}$ (10 to 15 larvae/ container). Larvae that stopped feeding on the diet and spun within $3 \mathrm{~d}$ of capture were regarded as "wandering", whereas those that fed actively and did not spin within $3 \mathrm{~d}$ were regarded as "feeding".
When these larvae reached the pre-pupal or pupal stage, they were placed in a plastic Petri dish (diameter, $9 \mathrm{~cm}$; height $2 \mathrm{~cm}$ ) and observed every day to check whether or not tachinid maggots had egressed from them (two individuals/dish). Tachinid maggots that egressed were placed into plastic Petri dishes (diameter, $5 \mathrm{~cm}$; height, $1 \mathrm{~cm}$ ) until adult emergence.

Among the tachinid species found at this study site, Drino inconspicuoides (Baranov) was easily identified at the maggot or puparial stage from the pair of spiracles at the end of the abdomen. Conversely, other tachinid species were often impossible to identify when they died before adult differentiation in the puparia. Hosts parasitized by such unidentified individuals were classified as "unknown".

Time of pupariation and winter survival in tachinids. To investigate the over-wintering status of tachinids, wandering host larvae of the third generation were collected in October 1995 and 1997 and placed with 10 pieces of layered tissue paper in a shallow plastic container $(26 \times 34 \times 5 \mathrm{~cm}$; 100 individuals/container). In 1997, wandering larvae captured on 17 and 28 October were separately incubated outdoors to investigate whether the time of sampling affected the over-wintering status and survival in tachinids. The containers were located in a shaded section of a veranda outside the laboratory. When the host larvae died or reached the pupal stage, they were individually transferred into small plastic vials (diameter, $1.5 \mathrm{~cm}$; height, $4 \mathrm{~cm}$ ) plugged with tissue paper. One to two hundred vials, each containing a single host, were placed onto moistened cotton in a plastic container $(26 \times 34 \times 5 \mathrm{~cm})$, and then kept continuously under ambient outdoor conditions until the following May. The container was checked at least once a month to determine whether tachinids had egressed from the host, pupariated or emerged as adults. In the following May, dead host pupae and live adults were dissected to check whether any tachinid maggots had died within the host body.

Over-wintering stage of Exorista japonica in hosts. To investigate the developmental stage of Exorista japonica (Townsend) over-wintering in host pupae, wandering host larvae were collected in October 1995 and kept under ambient outdoor conditions in a shallow plastic container $(26 \times 34 \times 5 \mathrm{~cm})$ with 10 pieces of layered tissue 
paper until pupation. These host pupae were placed in a plastic container (upper diameter, $5 \mathrm{~cm}$; bottom diameter, $4 \mathrm{~cm}$; height, $5 \mathrm{~cm}$; 20 pupae/container) and kept outdoors continuously. Because all D. inconspicuoides had egressed from their hosts by November, almost all tachinid maggots overwintering in the host pupae were E. japonica or Compsilura concinnata (Meigen). We can easily distinguish between host pupae parasitized by $E$. japonica and $C$. concinnata due to the presence (in the former species) or absence (in the latter) of a blackish hole that the maggot makes in the host's cuticle. Host pupae parasitized by E. japonica were harvested on 10 January, 6 February and 11 March 1996, and dissected to determine the developmental stage within the host. The tachinid maggot instar was judged by the morph and size of its pharyngeal skeleton.

Over-wintering survival of $\boldsymbol{E}$. japonica. To investigate when E. japonica pupae died in their puparia during over-wintering, tachinid puparia other than $D$. inconspicuoides, which were obtained from third-generation hosts during the period from 25 October to 10 December 1995, were placed in plastic Petri dishes (diameter, $9 \mathrm{~cm}$; height, $2 \mathrm{~cm}$ ) and kept outdoors continuously. They were transferred to the laboratory on either 15 December 1995, 10 January 1996 or 11 March 1996, and kept at room temperatures (approximately $25^{\circ} \mathrm{C}$ ) to check the rate of adult emergence. In addition, tachinid maggots that had egressed from hosts in late March of the following year were also transferred to the laboratory (on 15 April) to examine the rate of adult emergence. Emerged tachinid adults, except for $E$. japonica and unidentified pupae that had died before adult differentiation, were removed from these data.

Effect of environmental conditions on pupariation and adult emergence in $E$. japonica. To investigate whether photoperiodic conditions affect the timing of maggot egression from over-wintering hosts in E. japonica, over-wintering host pupae bearing the peculiar blackish mark and hole described above, which could have been parasitized by E. japonica, were transferred to laboratory conditions at 10 or $15^{\circ} \mathrm{C}$ under a $12 \mathrm{~L}: 12 \mathrm{D}$ (SD) or 16L : 8D (LD) photoperiod on 6 December 1996. These parasitized host pupae were observed daily until E. japonica maggots egressed.

To investigate whether photoperiodic conditions affect the timing of adult emergence from the overwintering puparia of E. japonica, the over-wintering tachinid puparia other than $D$. inconspicuoides were transferred to laboratory conditions at 10 or $15^{\circ} \mathrm{C}$ under a SD or LD photoperiod on 6 December 1996 . These puparia were observed daily until adult emergence. The emerged tachinid adults other than E. japonica and the unidentified puparia that had died before adult differentiation were removed from the data.

\section{RESULTS}

\section{Tachinid species and the rate of parasitization}

From 1995 to 1997, six species of tachinids were obtained from last instar $H$. cunea larvae at the described study site: E. japonica, Bessa parallera (Meigen), C. concinnata, D. inconspicuoides, Zenillia dolosa (Meigen) and Pales pavida (Meigen). The first two species are oviparous and the latter four ovoviviparous. In each generation of the host in each year, the total rate of parasitization by tachinids was significantly higher in wandering host larvae than in feeding ones $\left(\chi^{2}\right.$ test, $\left.p<0.01\right)$ (Fig. $1)$. The rate in wandering larvae was markedly higher in the third generation $(75,79$ and $70 \%$ in 1995, 1996 and 1997, respectively). E. japonica was the dominant species among the six tachinids in each generation in 1995 and 1997. In the third host generation in 1996, D. inconspicuoides and $C$. concinnata showed much higher rates of parasitization (46 and 24\%, respectively) than E. japonica $(2 \%)$.

In six cases, a pair of E. japonica and D. inconspicuoides emerged from a single third-generation host pupa. Gregarious parasitism, defined as when at least two adults of the same species were obtained from a single host pupa, was low in $E$. japonica (4, 0 and 1\% in 1995, 1996 and 1997, respectively) and in C. concinnata (0, 0 and 1\%), whereas it was relatively high in $D$. inconspicuoides, especially in the third host generation (65, 52 and $19 \%$ ).

The category "unknown" was mainly caused by under-development due to super parasitism (20 cases). No living or dead tachinid maggots were found throughout the experimental periods, not only in dead host larvae and pupae without visual marks or holes $(n=95)$, but also in emerged host adults $(n=212)$. No hyperparasitoids were obtained 


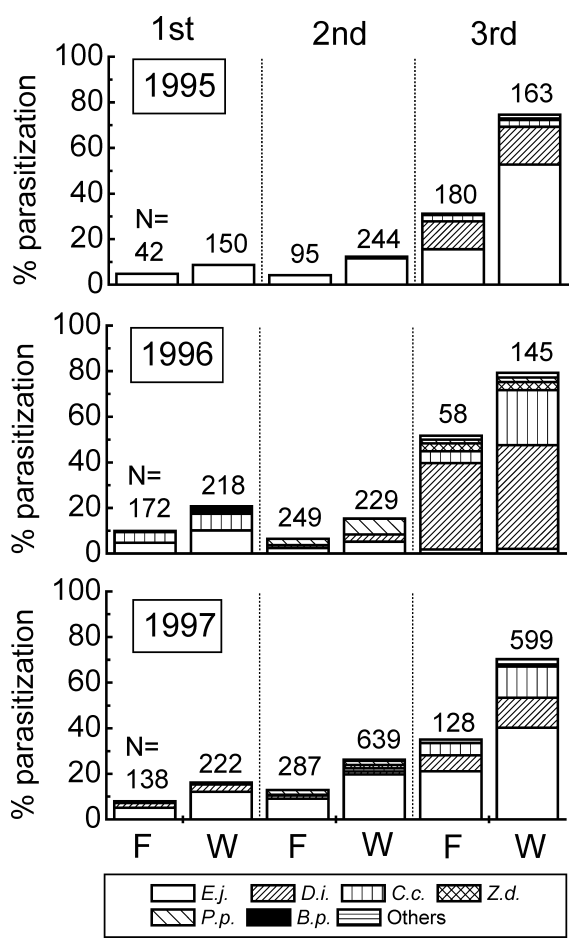

Fig. 1. Cumulative percentages of tachinid parasitism on feeding (F) and wandering (W) H. cunea larvae of the first to third generations in 1995, 1996, and 1997. The number of host larvae collected is shown in the figures. E.j., Exorista japonica; D.i., Drino inconspicuoides; C.c., Compsilura concinnata; Z.d., Zenillia dolosa; P.p., Pales pavida; B.p., Bessa parallela. "Others" includes the hosts parasitized by unidentified tachinids and those by E. japonica and D. inconspicuoides. The rate of parasitism by tachinids was significantly higher in wandering host larvae than in feeding ones $\left(\chi^{2}\right.$ test, $p<0.01$ ).

in this study.

\section{Time of pupariation and survival in tachinids}

The time of pupariation differed greatly among tachinid species parasitizing third-generation host larvae (Fig. 2). All D. inconspicuoides individuals pupariated until 10 November, and emerged as adults during November and December. C. concinnata and Z. dolosa egressed from the hosts and pupariated in the following February and March. On the other hand, two peaks of pupariation occurred in E. japonica: in late autumn to early winter (November and December) and the following early spring (March and April). In the first peak of pupariation, E. japonica maggots often egressed from dead host larvae and dead pre-pupae, and rarely from normally pupated hosts.

Irrespective of the collection dates, all individu-

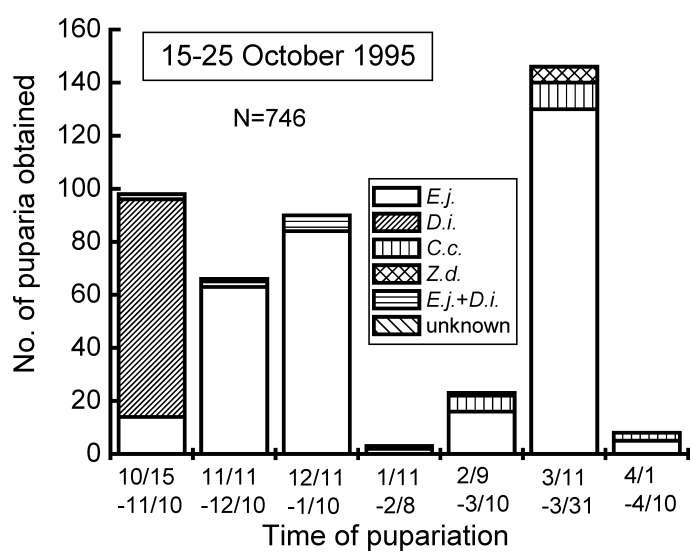

Fig. 2. Timing on pupariation of tachinids that egressed from the third generation of $H$. cunea collected from 15 to 25 October 1995. Each column shows the cumulative number of tachinids obtained. E.j., E. japonica; D.i., D. inconspicuoides; C.c., C. concinnata; Z.d., Z. dolosa. "Unknown" shows the hosts parasitized by unidentified tachinids.

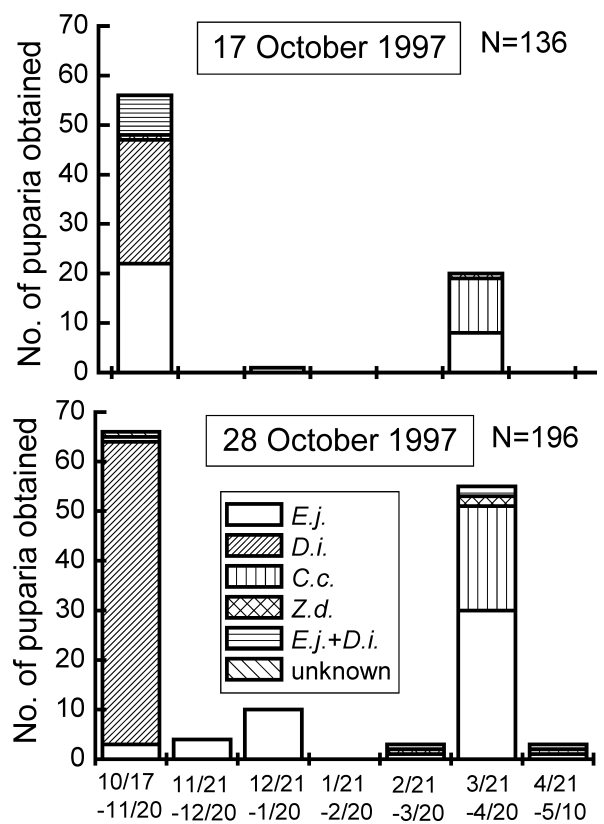

Fig. 3. Timing on pupariation of tachinids that egressed from the third generation of $H$. cunea collected on 17 and 28 October 1997. Each column shows the cumulative number of tachinids obtained. E.j., E. japonica; D.i., D. inconspicuoides; C.c., C. concinnata; Z.d., Z. dolosa. "Unknown" shows the hosts parasitized by unidentified tachinids.

als of $D$. inconspicuoides pupariated from 17 October to 20 November and those of $C$. concinnata pupariated from 21 March to 20 April (Fig. 3). There was no significant difference in the rate of adult emergence in D. inconspicuoides (72 and $79 \%$ on 17 and 28 October, respectively) or in $C$. concin- 


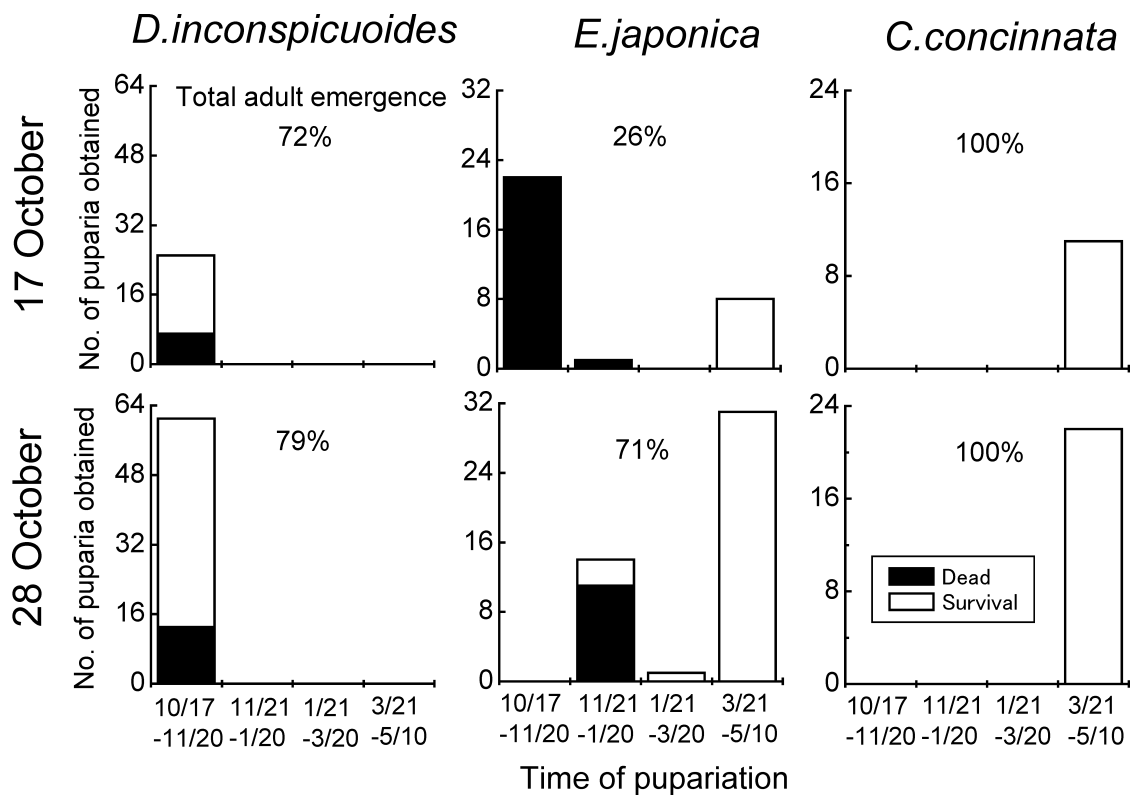

Fig. 4. Relationships between the timing of pupariation and the rate of adult emergence in D. inconspicuoides, E. japonica and C. concinnata obtained from the third generation of H. cunea collected on 17 and 28 October 1997. The rates of dead and adultemerged individuals are shown as black and white columns, respectively.

nata (100 and $100 \%)$ between the collection dates $\left(\chi^{2}\right.$ test, $p>0.1$, Fig. 4). On the other hand, $E$. japonica showed a different pattern in over-wintering status and survival between the two collection dates: $74 \%$ of individuals collected on 17 October pupariated in autumn or early winter, whereas only $32 \%$ of individuals collected on 28 October pupariated by early winter. The total rates of adult emergence in E. japonica were significantly higher when collected later $(26 \%$ on 17 October, $71 \%$ on 28 October; $\chi^{2}$ test, $p<0.01$ ). Almost all dead individuals had completed adult differentiation, but failed to egress from the puparia.

\section{Time of over-wintering death in $E$. japonica pupae}

Most E. japonica individuals that over-wintered at the puparial stage died before adult emergence (Fig. 4). Ninety percent of the individuals succeeded in normal adult emergence when transferred to the laboratory on 10 January, whereas $80 \%$ of the individuals failed to emerge as adults when transferred to the laboratory on 11 March (Fig. 5). All of the dead individuals transferred to the laboratory on both dates finished adult differentiation, but were not able to egress from their puparia. On the other hand, $96 \%$ of the individuals that had egressed from the host and pupariated in the following March succeeded in complete adult emergence.

\section{Over-wintering status of $E$. japonica in host pupae}

Seasonal changes in the developmental stages of

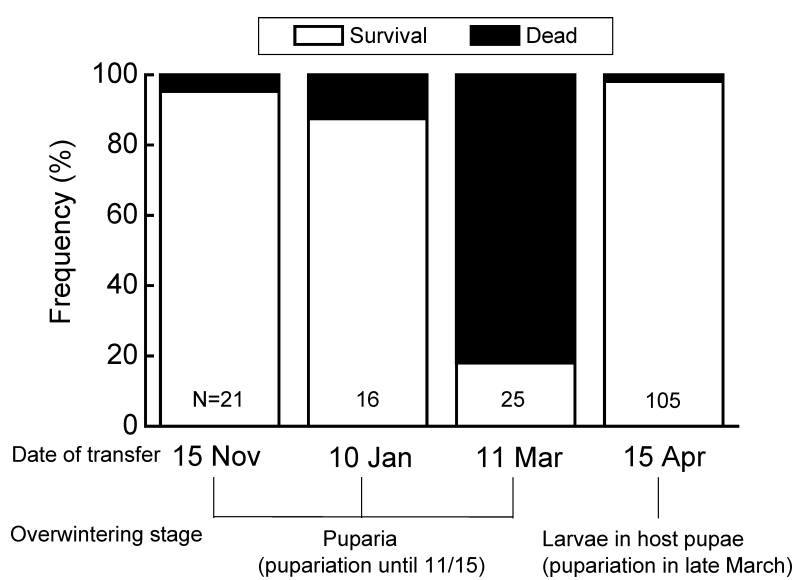

Fig. 5. Over-wintering survival of E. japonica puparia. Puparia obtained before 15 November were placed outdoors and transferred to the laboratory on 15 November, 10 January or 11 March. Individuals that over-wintered within the host pupae and egressed from the host in the following late March were also transferred to the laboratory on 15 April. The rates of dead and adult-emerged individuals are shown as black and white columns, respectively. The number of E. japonica puparia examined is shown in the figures. 


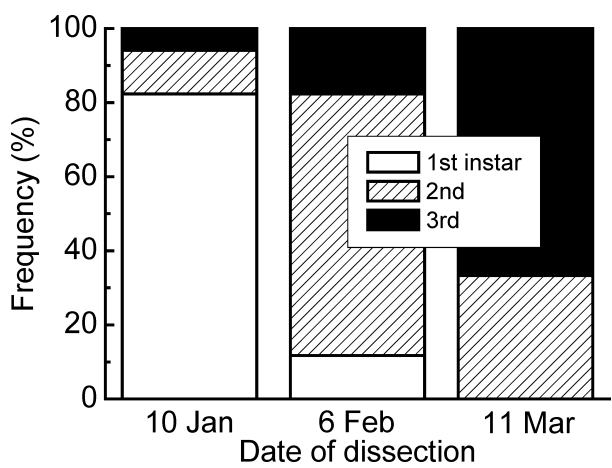

Fig. 6. Frequency of maggot stage of E. japonica overwintering in pupae of $H$. cunea. Over-wintering host pupae were dissected on 10 January, 6 February and 11 March. $N=30$ each.

E. japonica over-wintering within the host pupae were examined. On 10 January, more than $80 \%$ of the maggots were still at the first instar, whereas only 13 and $5 \%$ reached the second and third instars, respectively (Fig. 6). The proportion of later instar maggots gradually increased as the seasons progressed, and on 11 March no first instar maggots were found and $70 \%$ of the individuals had reached the third instar.

\section{Effect of environmental conditions on maggot egression and adult emergence in over-wintering E. japonica}

At both 10 and $15^{\circ} \mathrm{C}$, there were no significant differences between LD and SD photoperiods in the developmental period of E. japonica before maggot egression from the host pupae $\left(10^{\circ} \mathrm{C}\right.$ at LD, $n=12 / 25,84.1 \pm 13.8 \mathrm{~d} ; 10^{\circ} \mathrm{C}$ at SD, $n=11 / 25$, $90.0 \pm 20.6 \mathrm{~d} ; 15^{\circ} \mathrm{C}$ at $\mathrm{LD}, n=17 / 20,30.4 \pm 4.1 \mathrm{~d}$; $15^{\circ} \mathrm{C}$ at $\mathrm{SD}, n=15 / 20,32.4 \pm 3.9 \mathrm{~d}$; mean $\pm \mathrm{SE}, t-$ test, $p>0.1$ ). More than half of the host pupae died without maggot egression at $10^{\circ} \mathrm{C}(\mathrm{LD}, 52.0 \%$; SD, $56.0 \%$ ). When the dead host pupae were dissected 6 mo after their transfer to the laboratory, dead first instar maggots were frequently found inside the dead host pupae under both photoperiodic conditions (LD, 9 and 4 maggots at the first and third instars; SD, 11 and 3 maggots at the first and third instars).

There was also no significant difference between LD and SD photoperiods in the developmental period to adult emergence in over-wintering puparia $\left(10^{\circ} \mathrm{C}\right.$ at $\mathrm{LD}, n=7 / 22,82.8 \pm 6.0 \mathrm{~d} ; 10^{\circ} \mathrm{C}$ at $\mathrm{SD}$, $n=6 / 25, \quad 76.0 \pm 7.8 \mathrm{~d} ; \quad 15^{\circ} \mathrm{C}$ at $\mathrm{LD}, n=16 / 17$, $23.6 \pm 2.6 \mathrm{~d} ; 15^{\circ} \mathrm{C}$ at $\mathrm{SD}, n=15 / 17,24.5 \pm 2.0 \mathrm{~d} ; t$ - test, $p>0.1$ ). The puparial mortality was extremely high at $10^{\circ} \mathrm{C}$ irrespective of the photoperiod (LD, $68.1 \%$, SD, 76.0\%). All individuals completed adult differentiation in the puparia.

\section{DISCUSSION}

Parasitic wasps have been often used as biological control agents against insect pests and as research tools for elucidating the physiological and molecular mechanisms of parasitism. On the other hand, parasitic flies have received relatively little attention in general parasitoid ecology and physiology, even though Dipteran parasitoids are the second most abundant parasitic group after the Hymenoptera and include an estimated 16,000 species (Belshaw, 1994; Feener and Brown, 1997). The wider host range and difficulty of identification of parasitic flies might be considered as one of the reasons for this paucity of attention (Eggleton and Belshow, 1993; Belshow, 1994). The present study has demonstrated some of the over-wintering and parasitic strategies of polyphagous tachinids in a natural host and provides useful information for both fundamental and applied research.

In this study, six tachinid species were obtained from final instar $H$. cunea larvae in the period from 1995 to 1997. All species are parasitic on many lepidopteran species, including $H$. cunea (Shima, 1999). Among them, E. japonica, C. concinnata, B. parallela and P. pavida have an extremely wide host range: more than 40 species of lepidopteran hosts have so far been recorded (Shima, 1999). Conversely, no larval parasitic wasps were found in the $H$. cunea larvae at the study site used. This situation is probably related to the relatively narrow host range in hymenopteran parasitoids, especially in larval endoparasitic wasps (Eggleton and Belshaw, 1993; Feener and Brown, 1997). As generalists, tachinids were probably able to adapt much more rapidly to $H$. cunea, which invaded Japan only a half century ago, but specialist larval parasitic wasps might not have adapted yet in Japan (Hasegawa, 1966; Warren and Tadic, 1967). However, further study is needed to clarify parasitism by larval parasitic wasps in Japan because some larval parasitic wasps belonging to Apanteles or Meteorus, which use H. cunea as a host in North America, are distributed in Japan (Warren and Tadic, 1967). 
More than 40 species of tachinids have been reported as larval parasitiods of $H$. cunea throughout the world (Warren and Tadic, 1967, 1970), and 13 species in Japan (Shima, 1999). There are, however, only a few reports concerning seasonal changes in parasitization rate of tachinids in $H$. cunea. In first- and second-generation $H$. cunea pupae, the rate of parasitism by pupal parasitic wasps such as Pimla disparis is relatively high (10.0 to $37.0 \%$ ), but tachinids are rarely obtained (Sekiya, 1969; Takezawa et al., 1969; Tamura, 1969). The rate of parasitism by tachinids has been estimated at 14 to $25 \%$ in first- or second-generation host larvae (Konno, 1998) and about 7\% in those of the second generation (Kan et al., 2003). In the present study, the rate was less than $30 \%$ in the first and second generations of $H$. cunea, and extremely high in the third generation, especially in wandering larvae (70 to $80 \%$ ). Such a high rate of parasitism would affect the population size of the first generation of $H$. cunea in the following year.

The rate of parasitization on last instar host larvae was significantly higher at the wandering stage than at the feeding stage in each generation. All three dominant species, E. japonica, D. inconspicuoides and $C$. concinnata, which deposit their eggs directly on or into the host, showed similar tendency in the rates of parasitization, indicating that they preferably attack host larvae at the wandering stage. Indeed, E. japonica adults attacking wandering host larvae were often observed (Watanabe, personal observation). This ovipositional preference can be explained by the fact that host movement is an important factor for stimulating oviposition in E. japonica (Nakamura, 1997; Tanaka et al., 1999): wandering larvae move more actively than feeding ones.

There are few ecological studies of over-wintering status in tachinids. The present study clearly demonstrated different types of over-wintering strategy in tachinids using $H$. cunea as a host. $C$. concinnata over-wintered at the maggot stage in the host and E. japonica at the maggot and puparial stages. Conversely, all $D$. inconspicuoides individuals emerged as adults during November and December. The over-wintering stage of this tachinid species remains unknown. Because the tachinid is a polyphagous parasitoid (Shima, 1999), it might depend on other host species in late autumn and early winter after emergence from the third-genera- tion $H$. cunea host.

The two types of over-wintering found in the Tsukuba population of E. japonica were not observed in the Beijing population. All individuals of E. japonica egressed from $H$. cunea pupae in October, pupariated and over-wintered in the soil (Shi, 1981), although the timing of wandering in the third $H$. cunea generation is almost the same in Beijing and Tsukuba (October). Why the over-wintering status differs between the Tsukuba and Beijing populations of E. japonica should be the focus of future study.

Nakamura (1993) reported an effect of temperature on the development in E. japonica when it parasitizes the larvae of Pseudaletia separata. The lower thermal threshold of development is almost constant through egg to pupa (approximately $10^{\circ} \mathrm{C}$ ). In the present study, about half of the $E$. japonica maggots pupariated in autumn, whereas most of the rest remained as first instars until the following mid-January. If winter low temperatures simply delay maggot development, the second and third instar maggots should be found more often in over-wintering host pupae in January. Therefore, a portion of E. japonica larvae might delay their larval development through some regulation mechanism, such as diapause.

Several tachinids, Eurithia consobrina, Ceranthia samarensis and Athrycia cinerea, are known to enter pupal diapause in response to environmental conditions, such as photoperiod and temperature, irrespective of the host physiology (Wylie, 1977; Quednau, 1993; Turnock and Carl, 1995). On the other hand, in Pseudoperichaeta nigrolineata (=insidiosa) that enter diapause at the second maggot instar, host physiology is considered to be an important factor in the control of diapause (Grenier and Delobel, 1984; Ramadhane et al., 1988). In the present study, E. japonica tended to egress from the hosts of the third generation in autumn when hosts failed to pupate normally, whereas only a small number of E. japonica was obtained from normally pupated hosts in autumn. This probably indicates that early instar maggots of E. japonica stop their growth only when the host individuals of the third generation normally pupate and enter pupal diapause in autumn; host physiology thus seems to affect developmental arrest in E. japonica maggots. However, the timing of maggot egression from the host at relatively low temperatures (10 
and $15^{\circ} \mathrm{C}$ ) did not differ significantly between LD and SD photoperiods in E. japonica. How E. japonica regulates development during the maggot stage within a host and maintains two types of over-wintering strategy is still unknown.

Over-wintering survival rate differed greatly between the two types of winter status in E. japonica: individuals over-wintering at the maggot stage within hosts that almost succeeded in adult eclosion in the next year, whereas those over-wintering at the puparial stage out of hosts often died early in the following spring. E. japonica maggots overwintered more often within hosts collected in late October than those in mid-October. Thus, parasitizing host larvae in late autumn is advantageous for E. japonica when it uses $H$. cunea as a host.

The present study demonstrated that over-wintering strategy differs among tachinid species using $H$. cunea as a host. However, all the tachinid species reported in this study are generalists and could use other host species at any season including winter. For example, Nakamura (1993) calculated that E. japonica probably produces around six generations annually in this study area based on the effective cumulative temperature for development and the weather information,. However, the host $H$. cunea produces only two or three generations annually, indicating that E. japonica usually uses at least two host species within a single year. In 1996, when E. japonica was rarely obtained from the third generation of $H$. cunea, E. japonica should have used other host species during autumn and winter. Thus, tachinids as generalists can select their host species on a day-to-day basis, and the seasonal lifecycle of tachinids is largely different between species, between years, and probably between sites. The population dynamics of available host species also affects the lifecycle of tachinids. Further detailed ecological studies are necessary for understanding the complex lifecycle strategy of tachinid parasites.

\section{ACKNOWLEDGEMENTS}

I am grateful to S. Nakamura for critically reading the manuscript and useful information about tachinids, H. Shima and R. Ichiki for identification of tachinids and information in tachinid parasitism. I also thank T. Gomi for providing useful and important information about the lifecycle and rearing of H. cunea, S. Tanaka and T. Kotaki for helpful advice and encouragement and H. Ikeda, S. Ogawa and N. Kemmochi for laboratory assistance.

\section{REFERENCES}

Belshaw, R. (1994) Life history characteristics of Tachinidae (Diptera) and their effect on polyphagy. In Parasitoid Community Ecology (B. A. Hawkins and W. Sheehan eds.). Oxford University Press, New York, pp. 145-162.

Eggleton, P. and R. Belshaw (1993) Comparisons of dipteran, hymenopteran and coleopteran parasitoids: provisional phylogenetic explanations. Biol. J. Linn. Soc. 48: 213-226.

Feener, D. H., Jr. and B. V. Brown (1997) Diptera as parasitoids. Annu. Rev. Entomol. 42: 73-97.

Gomi, T. (1990) The transition to a trivoltine life cycle and mechanisms that enforce the voltism change in Hyphantria cunea Drury (Lepidoptera: Arctiidae). Appl. Entomol. Zool. 25: 483-489.

Gomi, T. (1996) Mixed life cycles in the transitional zone between voltisms in the fall webworm, Hyphantria cunea. Experientia 52: 273-276.

Gomi, T. (1997) Seasonal adaptation of a colonizing insect, the fall webworm, Hyphantria cunea Drury in Japan. Insectarium 34: 320-325 (in Japanese).

Grenier, S. and B. Delobel (1984) Determination of the arrest of larval development of the parasitoid Pseudoperichaeta insidiosa (Dipt., Tachinidae) in Galleria mellonella (Lepidopt., Pyralidae). Acta Oecol. Oecol. Appl. 5: 211-219 (in French).

Hasegawa, H. (1966) Some problems in the introduction and outbreak of the fall webworm, Hyphantria cunea Drury. Proc. Kanto-Tosan Plant Prot. Soc. 13: 5-16 (in Japanese).

Ito, Y., K. Miyashita and H. Yamada (1968) Biology of $H y$ phantria cunea Drury (Lepidoptera: Arctiidae) in Japan. VI. Effect of temperature on development of immature stages. Appl. Entomol. Zool. 3: 163-175.

Kan, E., N. Fukuhara and T. Hidaka (2003) Parasitism by tachinid parasitoids (Diptera: Tachinidae) in connection with their survival strategy. Appl. Entomol. Zool. 38: 131-140.

Kim, C. W. (1968) Study on the natural enemies proper in Korea attacking fall webworm, Hyphantria cunea Drury. Entomol. Res. Bull. (Seoul) 4: 37-55.

Konno, Y. (1998) Comparison of insecticide susceptibility between fall webworm, Hyphantria cunea (Lepidoptera: Arctiidae) and its parasitoid fly, Exorista japonica (Diptera: Tachinidae). Jpn. J. Appl. Entomol. Zool. 42: 167-170 (in Japanese with English summary).

Masaki, S. (1975) Biology of Hyphantria cunea Drury (Lepidoptera: Arctiidae) in Japan: a review. Rev. Plant Proect. Res. 8: 14-28.

Nakamura, S. (1993) Influence of temperature on development of Exorista japonica (Diptera: Tachinidae). Proc. Kanto-Tosan Plant Protect. Soc. 40: 207-208 (in Japanese).

Nakamura, S. (1997) Ovipositional behaviour of the parasitoid fly, Exorista japonica (Diptera: Tachinidae), in the laboratory: diel periodicity and egg distribution on a host. Appl. Entomol. Zool. 32: 189-195.

Quednau, F. W. (1993) Reproductive biology and laboratory 
rearing of Ceranthia samarensis (Villeneuve) (Diptera: Tachinidae), a parasitoid of the gypsy moth, Lymantria dispar (L.). Can. Entomol. 125: 749-759.

Ramadhane, A., S. Grenier and G. Plantevin (1988) Photoperiod, temperature and ecdysteroid influences on physiological interactions between diapausing Ostrinia nubilalis larvae and the tachinid parasitoid Pseudoperichaeta nigrolineata. Entomol. Exp. Appl. 48: 275-282.

Sekiya, J. (1969) On the emergence and the parasites in the first generation of the fall webworm. Proc. Kanto-Tosan Plant Protect. Soc. 16: 133-134 (in Japanese).

Shi, Y-S. (1981) Exorista japonica-a natural enemy of the fall webworm Hyphantria cunea Drury. Acta Entomol. Sin. 24: 342 (in Chinese).

Shima, H. (1999) Host-parasite catalog of Japanese Tachinidae (Diptera). MAKUNAGI, Suppl. 1: 1-108.

Takezawa, H., H. Asami and Y. Mizusawa (1969) Parasites on the second generation pupae of the fall webworm. Proc. Kanto-Tosan Plant Protect. Soc. 16: 134-136 (in Japanese).

Tamura, M. (1969) Notes on the parasitic insects of the fall webworm, Hyphantria cunea Drury. Proc. Kanto-Tosan Plant Protect. Soc. 16: 136-137 (in Japanese).
Tanaka, C., Y. Kainoh and H. Honda (1999) Physical factors in host selection of the parasitoid fly, Exorista japonica Townsend (Diptera: Tachinidae). Appl. Entomol. Zool. 34: 91-97.

Turnock, W. J. and K. P. Carl (1995) Evaluation of the Palaearctic Eurithia concobina (Diptera: Tachinidae) as a potential biocontrol agent for Mamestra configurata (Lepidoptera: Noctuidae) in Canada. Biocont. Sci. Technol. 5: 55-67.

Umeya, K. and Y. Ito (1977) Invasion and establishment of a new insect pest in Japan. In Adaptation and Speciation in the Fall Webworm (T. Hidaka ed.). Kodansha, Tokyo, pp. 1-12 (in Japanese).

Warren, L. O. and M. Tadic (1967) The fall webworm, Hyphantria cunea, its distribution and natural enemies: a world list (Lepidoptera: Arctiidae). J. Kans. Entomol. Soc. 40: 194-202.

Warren, L. O. and M. Tadic (1970) The fall webworm, Hyphantria cunea (Drury). Arkansas Agric. Exp. Stn. Bull. 759: 1-106.

Wylie, H. G. (1977) Preventing and terminating pupal diapause in Athrycia cinerea (Diptera: Tachinidae). Can. Entomol. 109: 1083-1090. 